\title{
Suelo-cemento-caliche como una solución sustentable a problemas geotécnicos
}

\section{Soil-cement-caliche as a sustainable solution to geotechnical problems}

\author{
Luis Apariciol, Manuel Campos ${ }^{l}$, Jenesis de León ${ }^{l}$, Traicy Guerra ${ }^{l}$, José Gallardo ${ }^{2 *}$ \\ ${ }^{1}$ Licenciatura en Ingeniería Civil - Facultad de Ingeniería Civil - Centro Regional de Chiriquí-Universidad Tecnológica de \\ Panamál ${ }^{I}$ \\ ${ }^{2}$ Facultad de Ingeniería Civil - Centro Regional de Chiriquí - Universidad Tecnológica de Panamá ${ }^{2 *}$
}

\begin{abstract}
Resumen El reciclaje de los residuos de concreto y suelos ayuda a reducir los efectos de contaminación debido a las construcciones. Se ha comprobado en diferentes estudios que la mezcla de residuos de concreto en rellenos es factible, de igual manera al incorporar cemento al suelo, ayuda a estabilizar las cimentaciones evitando asentamientos. El artículo analiza la posibilidad de utilizar suelocemento-caliche en diseños de mezclas para cimentaciones, muros de contención, capa base de carreteras, entre otros. Los resultados obtenidos en las muestras donde se reemplaza el agregado grueso por caliche arrojaron resultados donde el caliche tiende a aumentar la resistencia en los diseños de mezclas. Por otro lado, el reemplazo de los agregados finos y gruesos por suelo y caliche, tienden a aumentar la resistencia del diseño de suelo-cemento, convirtiendo estas mezclas en soluciones para el uso de estos residuos en las construcciones.
\end{abstract}

Palabras clave Suelo, cemento, caliche, flexión, compresión.

\begin{abstract}
The recycling of concrete and soil waste helps reduce the effects of pollution due to construction. It has been verified in different studies that the mixture of concrete residues in landfills is feasible, in the same way when adding cement to the ground, it helps to stabilize the foundations avoiding settlements. The article analyzes the possibility of using soil-cement-caliche in mixtures designs for foundations, retaining walls, base layer of roads, among others. The results obtained in the samples where the coarse aggregate is replaced by caliche yielded results where the caliche tends to increase the resistance in the mix designs. On the other hand, the replacement of fine and coarse aggregates by soil and caliche, tend to increase the resistance of the soil-cement design, converting these mixtures into solutions for the use of these residues in buildings.
\end{abstract}

Keywords Soil, cement, caliche, flexion, compression.

* Corresponding Author: jose.gallardo@utp.ac.pa

\section{Introducción}

El suelo es el material orgánico e inorgánico ubicado en la superficie de la tierra. Es considerado materia inerte, pero es capaz de dar vida debido a los compuestos químicos que la forman. El suelo es necesario para la agricultura, la minería y las actividades humanas. En la actualidad juega un papel importante en el área de la construcción, ya que el éxito de los proyectos se basa en los estudios preliminares que se realizan al suelo. Estos estudios proporcionan el espesor del suelo, la composición geológica, el nivel freático y la capacidad de resistencia. Estas características nos permiten conocer los tipos de cimentaciones que se adecuan al lugar.

En algunos casos el suelo donde se requiere realizar construcciones posee características desfavorables, esto conlleva al reemplazo o mejoramiento con un suelo favorable.
Una forma de mejorar el suelo es mezclándolo con cemento. La utilización de la mezcla suelo-cemento genera materiales estructurados artificialmente con tejido estable, esto debido a la presencia de enlaces artificiales y es por esto son utilizados como capas de base de carreteras, como también en pavimentos [1]. En los estudios realizados se dice que el suelo a pesar de sus características aislantes, inerciales y resistentes, son reducidas, debido a la humedad y se erosiona por acción de agentes externos [2], es por ello que empleando cemento, el cual constituye el medio estabilizante, esta mejora las condiciones del suelo respecto a la acción de agentes como la humedad, dándole características de estabilidad y resistencia [3]. Este uso de suelo tiene su justificación y la primera de ellas está en su extraordinaria abundancia: el $74 \%$ de la corteza 
terrestre es tierra: lo que determina su fácil y amplia disponibilidad y como consecuencia su bajo costo [4].

El objetivo general de la investigación es analizar la resistencia a compresión y flexión de mezclas de suelocemento-caliche, el cual se utiliza como un elemento estructural que da una solución geotécnica en muro de contención, estabilización del suelo y problemas de asentamientos en arcillas expansivas. En el 2015, Juan Manuel Quintero [5] determinó que al aumentar las proporciones de caliche tiene como consecuencia un aumento en la cantidad de cemento para cumplir con la resistencia esperada. Sin embargo, en otros estudios Piotr León Narlocha [6] demostró que se puede garantizar la capacidad de carga del dintel para una mezcla de suelo con $9 \%$ y cemento Portland modelado en el software FEM aplicado en paredes de vivienda de baja altura. Mientras que Jianguo Fan [7] observó que para obtener un mejor valor de cohesión hay que tener en cuenta la dosificación de cemento, como también el tipo de suelo, ya que la cohesión aumenta cuando la carga última incrementa, por lo que es un factor influenciado por muchos parámetros.

\section{Materiales y Métodos}

\subsection{Materiales}

Para el desarrollo de las pruebas de nuestro estudio elegimos materiales de uso común como lo son: caliche, cemento estructural tipo I, suelo, grava, arena, agua, moldes de vigueta y cilindros.

La figura 1 muestra algunos de los materiales y lugar de trabajo utilizado para realizar nuestra investigación.

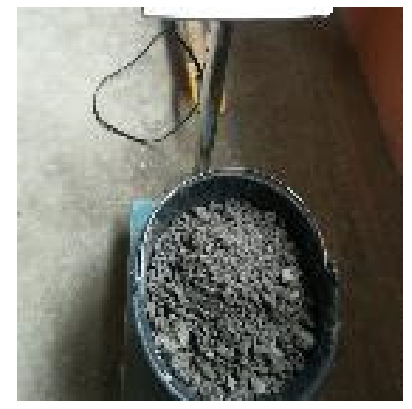

a) Caliche de Bloque

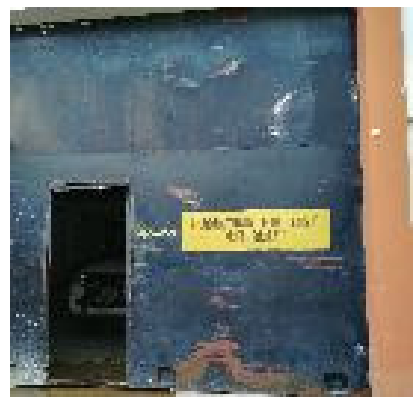

b) Laboratorio de suelos y materiales
Figura 1. Materiales, herramientas e instalaciones utilizadas.

\subsection{Diseño Experimental}

Para obtener las resistencias a compresión y flexión del suelo-cemento-caliche se aplicaron las normas ASTM C31, ASTM C39 y ASTM C78. Se planteó el uso de un patrón, es decir, una muestra, la cual será diseñada como concreto estructural para una resistencia de $28 \mathrm{MPa}$ e ir sustituyendo el agregando grueso y fino en tres proporciones.

De cada una de las mezclas se fabricaron dos cilindros y una vigueta, a los cuales se le realizaron las pruebas de resistencias a los siete días de edad, la nomenclatura se muestra en la tabla 1.
La tabla 2 muestra el valor de diseño del patrón para $1 \mathrm{~m}^{3}$ y la cantidad de concreto de agua, cemento, agregado grueso y fino, caliche y arcilla necesario para cada una de las pruebas.

La tabla 3, muestra las proporciones de los materiales para las mezclas P01, P02, P03 y P04.

\subsection{Programa experimental}

Para el desarrollo de la prueba realizamos los siguientes pasos:

\subsubsection{Obtención del caliche y del suelo}

Al ser el caliche un material reciclado producto de la demolición de estructuras, su composición es variante, por este motivo se tienen dos fuentes de caliche, una de bloques de construcción y el otro producto de la trituración de cilindros de concreto, a un tamaño máximo de 3/4".

Para el suelo utilizamos un tipo de arcilla a la cual le realizamos las pruebas granulométricas cuyos resultados se muestran en la tabla 4.

\subsubsection{Mezcla, proceso de vaciado y toma de espécimen}

Se realizó el mismo proceso para la mezcla de las cuatro pruebas, basados en las cantidades presentadas en la tabla 2.

Tabla 1. Nomenclatura y descripción de los cilindros y viguetas de las pruebas

\begin{tabular}{|c|c|}
\hline Nomenclatura & Descripción \\
\hline $\begin{array}{l}P 01-C 1 \\
P 01-C 2 \\
P 01-V 1\end{array}$ & $\begin{array}{l}\text { Cilindros y vigueta patrón, respectivamente. } \\
\text { Concreto diseñado para una resistencia a } \\
\text { compresión a los } 28 \text { días de curado de } 4 \mathrm{ksi} \text {. }\end{array}$ \\
\hline $\begin{array}{l}P 02-C 1 \\
P 02-C 2 \\
P 02-V 1\end{array}$ & $\begin{array}{l}\text { Cilindros y vigueta, respectivamente, con un } \\
\text { remplazo del } 50 \% \text { del agregado grueso del } \\
\text { patrón por caliche a razón de } 40 \% \text { de caliche } 3 / 4 \text { " } \\
\text { máximo y } 60 \% \text { de caliche bloque. }\end{array}$ \\
\hline $\begin{array}{l}P 03-C 1 \\
P 03-C 2 \\
P 03-V 1\end{array}$ & $\begin{array}{l}\text { Cilindros y vigueta, respectivamente, con un } \\
\text { remplazo del } 50 \% \text { del agregado grueso del } \\
\text { patrón por caliche a razón de } 40 \% \text { de caliche } 3 / 4 \text { " } \\
\text { máximo y } 60 \% \text { de caliche bloque y un } \\
\text { reemplazo del } 50 \% \text { de agregado fino del patrón } \\
\text { por arcilla. }\end{array}$ \\
\hline $\begin{array}{l}P 04-C 1 \\
P 04-C 2 \\
P 04-V 1\end{array}$ & $\begin{array}{l}\text { Cilindros y vigueta, respectivamente, con un } \\
\text { remplazo del } 100 \% \text { del agregado grueso del } \\
\text { patrón por caliche a razón de } 50 \% \text { de caliche } 3 / 4 \text { ", } \\
\text { máximo y } 50 \% \text { de caliche bloque y un } \\
\text { reemplazo del } 100 \% \text { de agregado fino del } \\
\text { patrón por arcilla. }\end{array}$ \\
\hline
\end{tabular}

Tabla 2. Cantidad de material necesario para realizar las mezclas de los cilindros y la vigueta de cada prueba

\begin{tabular}{|c|c|c|c|c|}
\hline Material & P01 (kg) & P02 $(\mathbf{k g})$ & P03 $(\mathbf{k g})$ & P04 (kg) \\
\hline Agua & 7.47 & 7.47 & 7.47 & 7.47 \\
\hline Cemento & 13.11 & 13.11 & 13.11 & 13.11 \\
\hline $\begin{array}{c}\text { Agregado } \\
\text { Grueso }\end{array}$ & 38.96 & 19.48 & 19.48 & 0.000 \\
\hline $\begin{array}{c}\text { Agregado } \\
\text { Fino }\end{array}$ & 25.85 & 25.85 & 12.93 & 0.000 \\
\hline
\end{tabular}


Aparicio (et al): Suelo-cemento-caliche como una solución sustentable a problemas geotécnicos

\begin{tabular}{|c|c|c|c|c|}
\hline Caliche & 0.000 & 19.48 & 19.48 & 38.96 \\
\hline Arcilla & 0.000 & 0.000 & 12.93 & 25.39 \\
\hline $\begin{array}{c}\text { Caliche } \\
\text { 3/4 }\end{array}$ & 0.000 & 7.79 & 7.79 & 12.86 \\
\hline $\begin{array}{c}\text { Caliche } \\
\text { Bloque }\end{array}$ & 0.000 & 11.69 & 11.69 & 12.86 \\
\hline
\end{tabular}

Tabla 3. Dosificación de materiales en porcentajes

\begin{tabular}{|c|c|c|c|c|}
\hline Material & P01 (\%) & P02 (\%) & P03 (\%) & P04 (\%) \\
\hline Agua & 9 & 9 & 9 & 9 \\
\hline Cemento & 15 & 15 & 15 & 15 \\
\hline $\begin{array}{c}\text { Agregado } \\
\text { Grueso }\end{array}$ & 46 & 23 & 23 & 0 \\
\hline $\begin{array}{c}\text { Agregado } \\
\text { Fino }\end{array}$ & 30 & 30 & 15 & 0 \\
\hline Caliche & 0 & 23 & 23 & 46 \\
\hline Arcilla & 0 & 0 & 15 & 30 \\
\hline
\end{tabular}

Table 4. Resultados de la Granulometría de la Arcilla y Caliche de Bloques

\begin{tabular}{|l|c|c|}
\hline \multirow{2}{*}{ \# Tamiz } & \multicolumn{2}{|c|}{ \% Que pasa } \\
\cline { 2 - 3 } & Arcilla & $\begin{array}{c}\text { Caliche } \\
\text { Bloque }\end{array}$ \\
\hline $3 / 8 ”$ & - & 94.36 \\
\hline$\# 4$ & 91.58 & 61.01 \\
\hline$\# 8$ & - & 37.30 \\
\hline$\# 10$ & 67.75 & - \\
\hline$\# 16$ & - & 23.29 \\
\hline$\# 20$ & 44.79 & - \\
\hline$\# 30$ & - & 14.55 \\
\hline$\# 40$ & 23.65 & - \\
\hline$\# 50$ & - & 9.67 \\
\hline$\# 60$ & 11.56 & - \\
\hline$\# 100$ & 3.04 & 6.16 \\
\hline$\# 200$ & 0.11 & 2.63 \\
\hline
\end{tabular}

\subsubsection{Proceso de desencofrado y curado}

Los cilindros y viguetas ya vaciados con sus respectivas mezclas, los cubrimos y procedimos a desencofrarlos a las 46 horas.

Posteriormente los colocamos en la tina con agua y cal, hasta cumplir la edad de 7 días.

\subsubsection{Pruebas de flexión y compresión}

Para realizar las pruebas se siguieron los pasos establecidos por las normas ASTM C31, ASTM C39 y ASTM C78.

Para el cálculo del módulo de rotura de las viguetas utilizamos la ecuación (1) señalada en la Norma ASTM C78.

$$
R=\frac{P L}{b d^{2}}
$$

Donde:

$R=$ Módulo de rotura, $\frac{\mathrm{kg}}{\mathrm{cm}^{2}}$

$P=$ carga máxima aplicada indicada por la máquina de prueba, $k g$.

$L=$ Longitud entre apoyo, $45 \mathrm{~cm}$.

$d=$ profundidad promedio de la muestra, $\mathrm{cm}$.

$b=$ ancho promedio de la muestra, $\mathrm{cm}$.

En la figura 3 se muestra las fallas presentadas en los diferentes cilindros de pruebas.

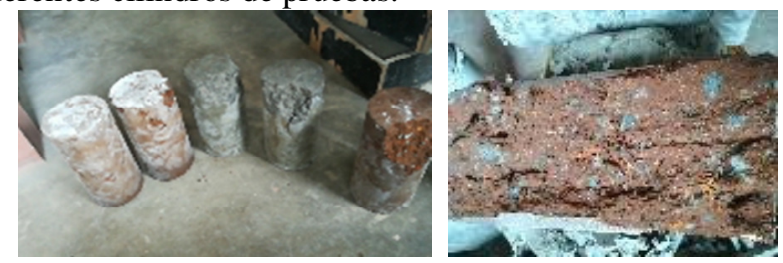

a) Fallas en los cilindros

b)Falla presentada en $\mathrm{P} 03-\mathrm{C} 2$

Figura 2. Cilindros después de realizada la prueba.

\section{Materiales y métodos \\ 3.1 Resultados}

Las pruebas se realizaron según lo establecido en el programa experimental, obteniéndose los resultados de las pruebas de compresión y flexión a los siete días de curado, dichos resultados son mostrados en la tabla 5 y tabla 6 respectivamente. Para el cálculo de la resistencia a compresión se tiene un área de cilindro de $176.71 \mathrm{~cm}^{2}$.

Tabla 5. Resistencia a compresión a los 7 días de curado

\begin{tabular}{|l|l|c|c|}
\hline Prueba & $\begin{array}{l}\text { Carga } \\
\text { Total (kg) }\end{array}$ & $\begin{array}{c}\text { Resistencia a } \\
\text { Compresión } \\
\left(\frac{\boldsymbol{k g}}{\boldsymbol{c m}^{2}}\right)\end{array}$ & $\begin{array}{c}\text { Resistencia a } \\
\text { Compresión } \\
(\mathbf{k s i})\end{array}$ \\
\hline P01-C1 & 32304.18 & 182.8 & 2.6 \\
\hline P01-C2 & 32304.18 & 182.8 & 2.6 \\
\hline P02-C1 & 35004.18 & 198.1 & 2.8 \\
\hline P02-C2 & 36404.18 & 206.0 & 2.9 \\
\hline P03-C1 & 18404.18 & 104.1 & 1.5 \\
\hline P03-C2 & 18404.18 & 104.1 & 1.5 \\
\hline P04-C1 & 14204.18 & 80.4 & 1.1 \\
\hline P04-C2 & 14104.18 & 79.8 & 1.1 \\
\hline
\end{tabular}

Para realizar el cálculo del módulo de rotura se utilizó la ecuación (1) y los resultados se muestran en la tabla 6 .

Tabla 6. Módulo de Rotura a los 7 días de curado

\begin{tabular}{|l|c|c|c|c|c|}
\hline \multirow{2}{*}{ Prueba } & \multirow{2}{*}{$\begin{array}{c}\text { b } \\
\text { (pulg) }\end{array}$} & $\begin{array}{c}\text { d } \\
(\mathbf{p u l g})\end{array}$ & $\begin{array}{c}\text { Carga } \\
\text { Total (lb) }\end{array}$ & \multicolumn{2}{|c|}{ Módulo de rotura } \\
\cline { 4 - 6 } & & & & $\mathrm{Kg} / \mathrm{cm}^{2}$ & Psi \\
\hline$P 01-V 1$ & 6.1 & 6.1 & 3279.56 & 18.29 & 260.08 \\
\hline$P 02-V 1$ & 6.1 & 7.5 & 8889.08 & 32.78 & 466.31 \\
\hline$P 03-V 1$ & 6.1 & 6.3 & 4479.08 & 23.41 & 333.00 \\
\hline$P 04-V 1$ & 6.1 & 6.1 & 4479.08 & 24.97 & 355.20 \\
\hline
\end{tabular}

\subsection{Análisis de Resultados}

De los resultados mostrados, se puede observar que el comportamiento es algo de lo esperado, a medida que 
aumentamos la proporción de suelo en la mezcla, el resultado a compresión ha ido disminuyendo.

En la prueba patrón P01 notamos que el valor ideal de un concreto normal da una resistencia a los siete días de curado de $183 \frac{\mathrm{kg}}{\mathrm{cm}^{2}}$.

La P03 alcanzó una resistencia de $104.1 \frac{\mathrm{kg}}{\mathrm{cm}^{2}}$, un valor por debajo del patrón sin embargo está dentro del rango de un concreto pobre de $91.39 \frac{\mathrm{kg}}{\mathrm{cm}^{2}}$, y la P04 queda debajo de este valor con una resistencia de $80.1 \frac{\mathrm{kg}}{\mathrm{cm}^{2}}$.

El suelo-cemento tiene una resistencia de $74 \frac{\mathrm{kg}}{\mathrm{cm}^{2}}$ [1], la P04 muestra un claro incremento en la resistencia al incorporar el caliche y esta diferencia es aún más notoria en P03.

Cuando el patrón es comparado con la P02 se aprecia un aumento en la resistencia del concreto hasta $202 \frac{\mathrm{kg}}{\mathrm{cm}^{2}}$, demostrando que el caliche es una muy buena forma de mejorar el concreto, que es un resultado constante en otras investigaciones [8], [9]. Este resultado se debe primordialmente, a la capacidad de absorción que posee el caliche haciendo que la relación agua cemento disminuya y así arrojar una resistencia mayor [10].

La P01 y P02, mantenían una relación $\frac{A}{C}=0.57$ con lo cual debe arrojar una resistencia de $183 \frac{\mathrm{kg}}{\mathrm{cm}^{2}}$ a los siete días, que es lo obtenido en P01. Sin embargo, para P02 la resistencia es de $202 \frac{\mathrm{kg}}{\mathrm{cm}^{2}}$ que no corresponde a su relación $\mathrm{A} / \mathrm{C}$, lo que nos lleva a pensar que la capacidad de absorción del caliche disminuyó esta relación. Las correcciones de humedad y absorción no fueron realizadas para el caliche, las cuales tenía $4.48 \%$ y 8.67 $\%$ respectivamente, arrojando una corrección de agua de 0.49 $\mathrm{kg}$. Esto baja la relación agua cemento a $\frac{A}{C}=0.532$, lo cual corresponde a una resistencia de $199 \frac{\mathrm{kg}}{\mathrm{cm}^{2}}$, lo cual explica de gran manera estos resultados. A pesar de ello deja la puerta abierta para tomar en cuenta otros factores como la presencia de cemento en el caliche y el proceso de endurecimiento del concreto en el tiempo.

Al observar la tabla 6 los resultados muestran un módulo de rotura mayor para $\mathrm{P} 02, \mathrm{P} 03, \mathrm{P} 04$, al compararlos con $\mathrm{P} 01$; lo cual muestra que, al agregar suelo y caliche a la mezcla, se incrementa la resistencia máxima en la fibra.

\section{Conclusiones}

Obtenidos los resultados y haciendo un análisis de ellos, tenemos las siguientes conclusiones:

- Estos resultados son una muestra clara de la capacidad del caliche para ser un material que aporta resistencia al suelocemento y aún más al concreto regular, lo cual reduce la cantidad de materiales no renovables necesarios para la mezcla de concreto.

- Si se comparan las pruebas P03 y P04, al agregar mayor cantidad de suelo y caliche reemplazando el agregado fino y grueso, respectivamente, del $50 \%$ al $100 \%$, la resistencia a compresión va a ir disminuyendo hasta aproximadamente un $27 \%$, como se muestra en la tabla 5 .

- La mezcla de cemento-caliche dio muy buenos resultados, donde al mantener la relación $\mathrm{A} / \mathrm{C}$, se puede obtener por mínimo, la misma resistencia que del concreto normal, permitiendo al caliche ser un sustituto de los agregados no renovables.

- Esta investigación deja el camino abierto para realizar más estudios sobre los aportes a la resistencia del caliche al concreto y a mezclas de suelo-cemento, para encontrar de una vez por todas una solución a este tipo de desperdicios.

- La arcilla disminuye la resistencia.

\section{RECOMENDACIONES}

Basados en la tabla 3, donde se muestra las proporciones de los materiales para la mezcla de suelo-cemento-caliche, recomendamos las siguientes aplicaciones:

- Utilizar las proporciones de la P03 como un sustituto ideal para el concreto de 2 ksi o concreto de baja resistencia.

- En las cimentaciones se suele utilizar un concreto de $2 \mathrm{ksi}$, de alrededor de 5 a $10 \mathrm{~cm}$ de espesor, sobre el cual se inicia el vaciado de las cimentaciones, este concreto puede ser remplazado por las proporciones de la $\mathrm{P} 03$.

- Para cualquier uso donde se requiera un material con una resistencia a compresión menor a 1.7 ksi, se recomienda la proporción P04.

- Para la capa base la resistencia se encuentran entre 20 y 100 $\frac{\mathrm{kg}}{\mathrm{cm}^{2}}$ [11], para el cual la proporción P03 es una solución viable.

- Para el diseño estructural de muros con un concreto de 4 ksi, se recomienda la proporción P02.

\section{AGRADECIMIENTOS}

Agradecemos al Lcdo. Arístides Perén y al Ing. Pedro Aparicio por su colaboración, y contribuir con sus conocimientos y tiempo a nuestra investigación. También por permitirnos el acceso al Laboratorio de suelos y materiales, y todas las herramientas y equipo que requerimos.

\section{REFERENCIAS}

[1] Daniel Ribeiro, Raquel Néri, and Rafaela Cardoso. Influencia del contenido de agua en el UCS de mezclas suelo-cemento para diferentes dosis de cemento. Advances in Transportation Geotechnics 3. The 3rd International Conference on Transportation Geotechnics (ICTG 2016).

[2] Barros, L. P., et al. (2010). Resistencia sísmica del suelocemento postensado en construcciones de baja complejidad geométrica.

[3] Mariana P. Gatani (2000). Ladrillos de suelocemento:mampuesto tradicional en base a un material sostenible. 
[4] José Toirac Corral. (2008). El suelo-cemento como material de construcción.

[5] Juan Manuel Quintero Esquivel (2017). Suelo-cemento con sustitución en franja granulométrica con concreto reciclado.

[6] Piotr Leon Narlocha, et al. (2015). Flexural tensile strength of construction elements made of cement stabilized rammed earth.

[7] Jianguo Fan, Dongyuan Wang, Duo Qian (2018). Soil-cement mixture properties and design considerations for reinforcedexcavation. Journal of Rock Mechanics and Geotechnical Engineering xxx.

[8] Dominguez Lepe. J. A. y Martínez L. Emilio (2007). Reinserción de los residuos de construcción y demolición al ciclo de vida de la construcción de viviendas.
[9] Ángela María Hincapié Henao, Elisa Andrea Aguja López (2003). Agregado reciclados para morteros.

[10] I.E. Martínez-Soto, C.J. Mendoza-Escobedo (2005). Comportamiento mecánico de concreto fabricado con agregados reciclados.

[11] Mario Arce (2011). Bases estabilizadas con cemento, algunos comentarios sobre sus ventajas e inconvenientes. Boletín Técnico PITRA. 